\title{
Transposable elements contribute to fungal genes and impact fungal lifestyle

\section{Anna Muszewska ${ }^{1, * \#, ~ K a m i l ~ S t e c z k i e w i c z ~}{ }^{2, \#}$, Marta Stepniewska-Dziubinska ${ }^{1}, K^{\prime} z y s z t o f$ Ginalski² \\ ${ }^{1}$ Institute of Biochemistry and Biophysics, Polish Academy of Sciences, Pawinskiego 5A, 02-106 Warsaw, Poland 2 University of Warsaw, CeNT, Laboratory of Bioinformatics and Systems Biology, Zwirki i Wigury 93, 02-089, Poland *musze@ibb.waw.pl \\ \#These authors contributed equally and should be considered as joint first authors}

Introduction: Transposon insertion can have deleterious consequences for target loci via a range of different scenarios. In the last decade remarkable examples of functional impact of transposons on host fate have been described. TE neighborhood has been shown to repress neighboring genes in fungal taxa. Encouraged by experimental screenings showing impact of TEs on gene expression we performed a systematic analysis of genomic location of TEs in 632 publicly available fungal genomes from NCBI database.

Results: We found that non autonomous transposons and remnants massively overlap with regions annotated as genes. These results suggest a great contribution of transposon derived sequences to host's genes (Fig.1). The overall ubiquity of TEs neighboring genes can be a sign of neutrality of old, fragmented TEs in gene proximity, the lack of traceable signs of target site preference among most types of TEs and the recurrent usage of TE-derived sequences. Younger, potentially active transposons cluster together with other transposons. Observed non random distribution of potentially active transposons might be a sign of selection against insertion of TE in gene proximity and target site preference among some types of TEs. The ubiquity of transposon fragments even in areas devoid of functional TEs does not support the hypothesis of insertion bias (omitting genes) as a key player shaping TE genomic placement.

Proteins coded by genes impacted by old transposon insertions have significantly less repeat motifs and protein-protein interaction domains (eg. RRM and Ank) but are more abundant in enzymatic domains (Glyco_transf_28, Cu-oxidase, Catalase, Aconitase and ATP-synthase).

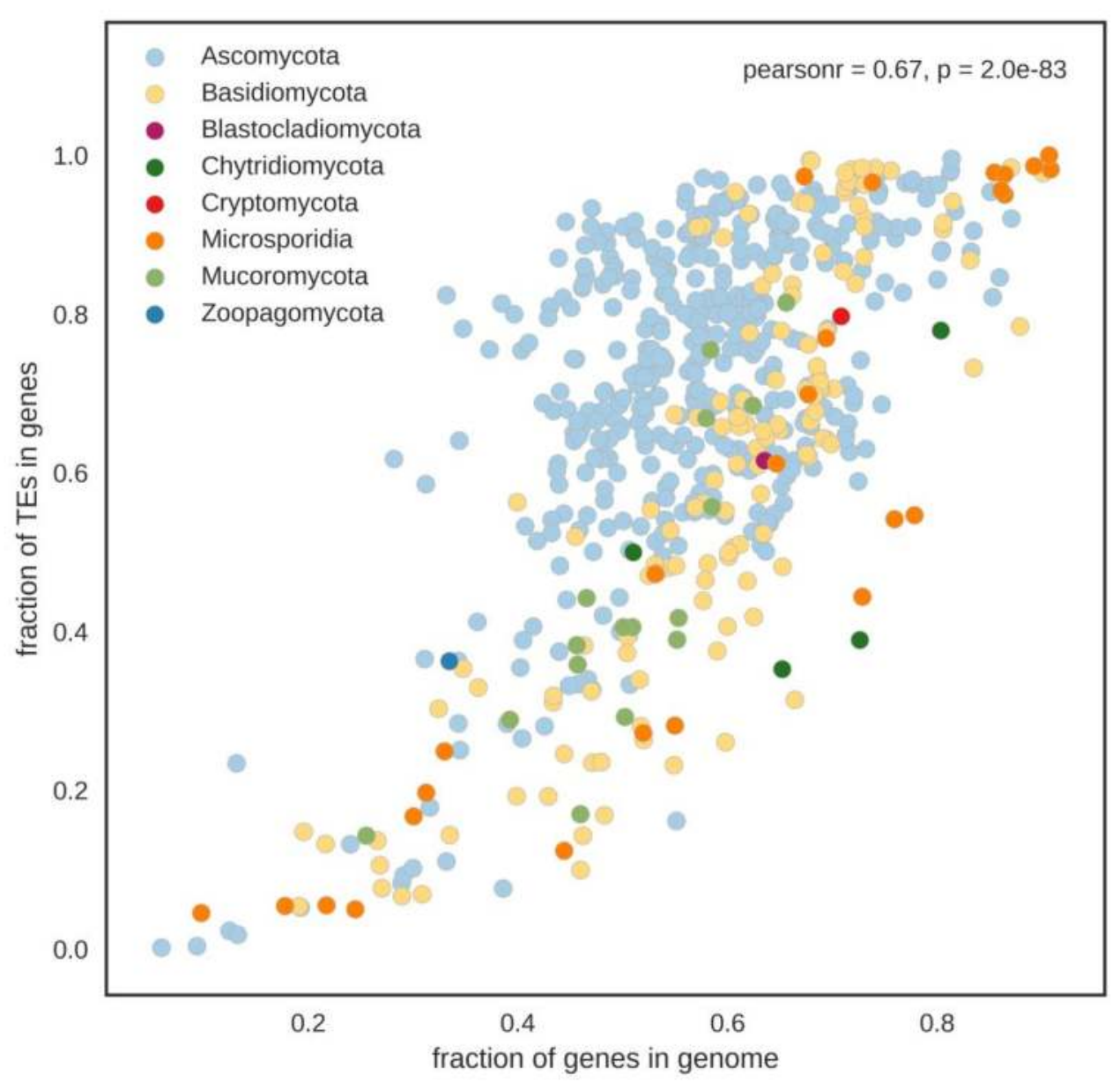

Fig 1. Distribution of in-gene TEs without transposon-related domains in genomes with different gene density.
Animal related and pathogenic fungi have more TEs inserted into genes than fungi with different lifestyles (Fig. 2). Plant associated, symbiotic and saprotrophic fungi have less in-gene TEs than other fungi. The overlapping of transposon derived sequences with genes is particularly common in taxa with compact genomes. Our findings show a link between the mobilome and fungal ecology.

Observed localization of transposons in 632 fungal genomes shows a dichotomy between relatively young elements depleted in genes compared to sequences clearly derived from transposons. This phenomenon points at a likely scenario of domestication of TEs and utilization as evolutionary raw material for selection.
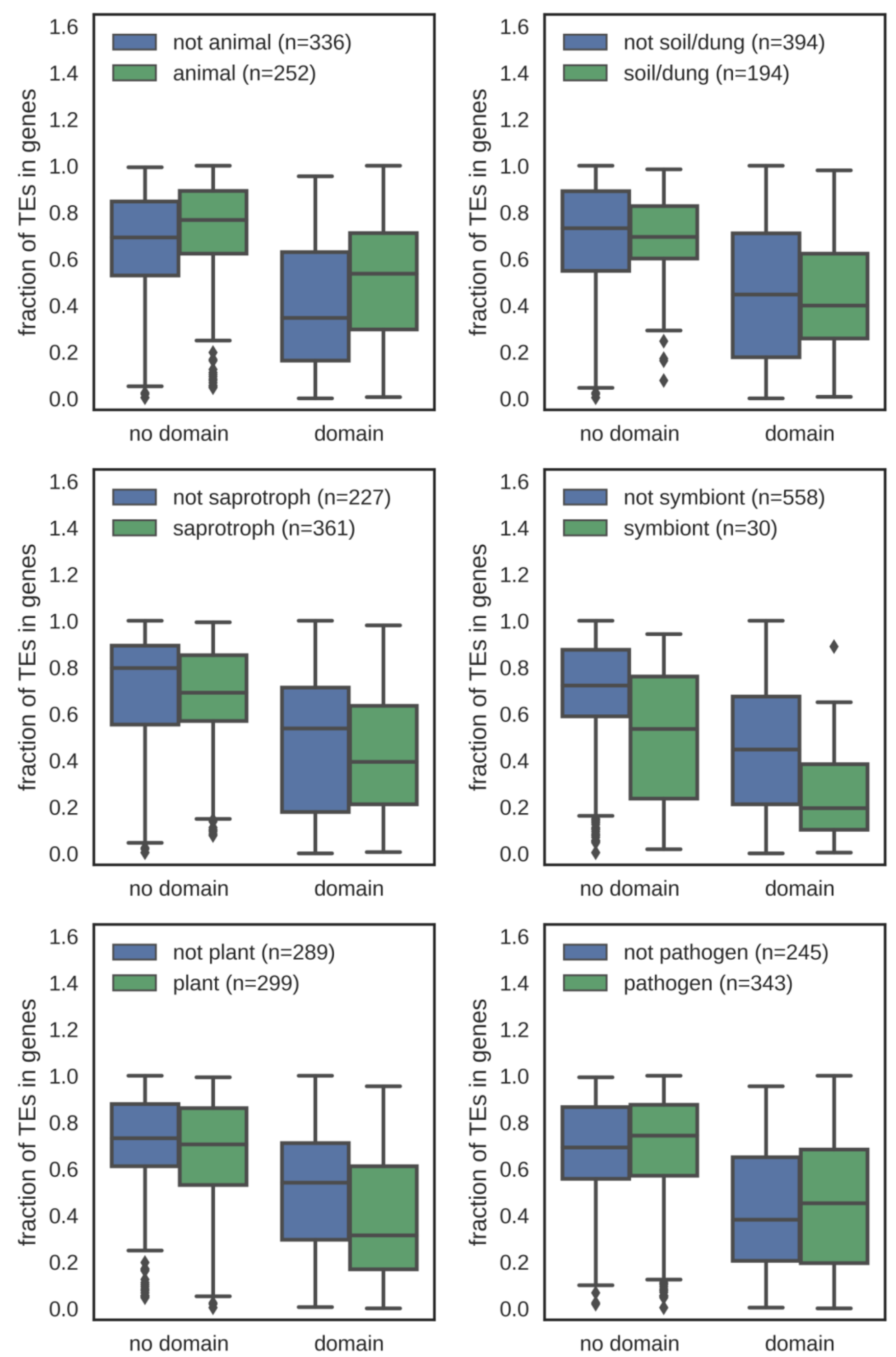

Fig 2. Distribution of TEs in fungi with different ecological features. 